\title{
COMPARISON OF TEMPERATURE AND MECHANICAL STRESS-STRAIN DISTRIBUTIONS OF STEEL BILLETS WITH SHARP AND ROUNDED CORNERS
}

\author{
Michal BŘEZINA, Tomáš NÁVRAT, Josef ŠTĚTINA, Jaroslav KOVÁŘ, Lubomír KLIMEŠ, \\ Tomáš MAUDER, Jindřich PETRUŠKA
}

Brno University of Technology, Technická 2, 61669 Brno, Czech Republic, EU, Michal.Brezina1@vutbr.cz

https://doi.org/10.37904/metal.2020.3443

\begin{abstract}
Monitoring and evaluation of temperature distribution in continuous steel casting coupled with optimal temperature intervals providing no defects can be used for the prediction of crack formation in the cast strand. Crack locations can be further specified with the use of mechanical stress-strain distribution model. These crack locations are strongly dependent on the zero ductility temperature (ZDT) and the liquid impenetrable temperature (LIT). To avoid the excessive stress and strain distribution in the steel, parts of the strand where bending and unbending take place should not be in ZDT and LIT intervals. Thus, the casting speed and water flow rates through cooling nozzles have to be modified to avoid these crack sensitive temperature intervals where the stress exceeds its maximum allowable value determined from the crack criteria. In this paper, the thermal solidification and mechanical stress-strain distribution models of billets with sharp and rounded corners for structural steel grades S355 are presented and the results are compared. Further, the idea of coupling between thermal and mechanical models is presented, which serves as a base for a crack predictive model assessing the quality of cast semi-products.
\end{abstract}

Keywords: Continuous casting, thermal model, mechanical model, crack formation, quality prediction

\section{INTRODUCTION}

The aim of scientists and researchers in the continuous casting (CC) field is to increase the quality of final products in order to fulfil challenging demands on surface quality as are in automotive, space, offshore industries. To prevent cracks to occur, developing of the temperature field in the strand through the whole casting process has to be known and the casting parameters for particular grades of steel must be set. This can be done by appropriate adjustments in primary (mould) cooling zone and in secondary cooling zone in terms of accurately calculated cooling intensities beneath nozzles, rollers and precise radiation to open space and into other parts of the continuous casting machine (CCM). It is known that the steel of a defined chemical composition is prone to cracking under certain circumstances. In order to determine crack sensible locations, models describing the temperature distribution of the strands have been of great importance and helped researchers to answer their questions about the temperature intervals, which guarantee the strand with minimum or even no defects for specified grade of steel, see [1]. The fully developed 3D Brno Dynamic Solidification Mode ${ }^{\circledR}$ (BrDSM) [1] was created at Brno University of Technology a few years ago. Based on the calculated temperature fields and known crack sensible temperature intervals, it can be concluded if the casting parameters are set well or not. The BrDSM model was designed to not just monitor temperature fields but also to make some regulations in terms of adapting the casting speeds and water flow rates in cooling airmist nozzles to ensure predefined temperatures at specified locations. With great number of quality records from the castings the model can predict the quality based on the temperature distribution only. But this approach is not sufficient and mechanical properties, which affect the strand, have to be considered as well. Monitoring of the mechanical stress in the whole strand gives other information, which is crucial in investigating the quality of cast semi-products [2]. The locations where the stress and strain distribution exceeds the 
maximum allowable values are especially in bending and unbending parts of the CCM in mushy zone near the solid state where the steel is most prone to cracking due to the zero ductility temperature and liquid impenetrable temperature [3]. In this article the primary effort is to develop thermal and mechanical models, which can be coupled together in terms that they will enable a real time accurate prediction of crack formation in the strand. Some assumptions and modifications are done in the thermal model and the $3 \mathrm{D} \mathrm{BrDSM}$ is degraded to the so-called 2.5D slice model as depicted in Figure 1. This step is of great importance while heat transfer in the casting direction does not influence the overall temperature distribution in great measures. On the other hand, this approach enables the real time calculation even in the case when the mechanical model is influenced by the thermal model and evaluations of mechanical stress distributions have to be done repeatedly. The reliability of both models is verified on computation of the temperature and mechanical stressstrain distribution of billets with sharp corners (SC) and rounded corners (RC). The corner cracks occur due to the excessive cooling in primary and secondary cooling zones resulting in large thermal gradients and evoke intense stress which yields for corner types of deformation [4]. The results are then compared and discussed.

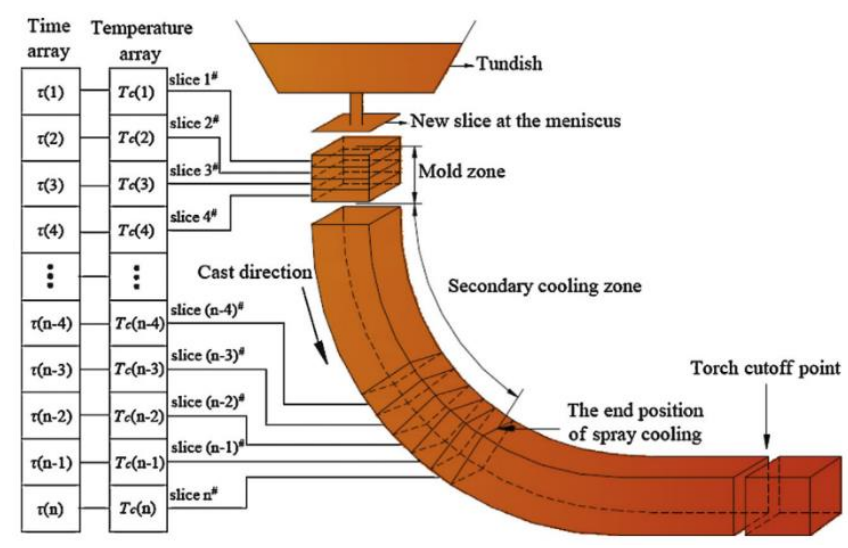

Figure 1 Construction idea of 2.5D - slice model [5]

\section{STEEL GRADE}

A structural steel grade S355 is used in this work for which thermal and mechanical properties are calculated. The steel grade S355 has $0.23 \%$ wt. carbon content and other alloying elements such as manganese, phosphor, chromium, etc. To receive accurate varying properties of particular steel grade in a whole descending temperature range, the use of the solidification analysis package IDS [6] (interdendritic solidification software) is necessary. The IDS is more comfortable and practical to use than expensive and
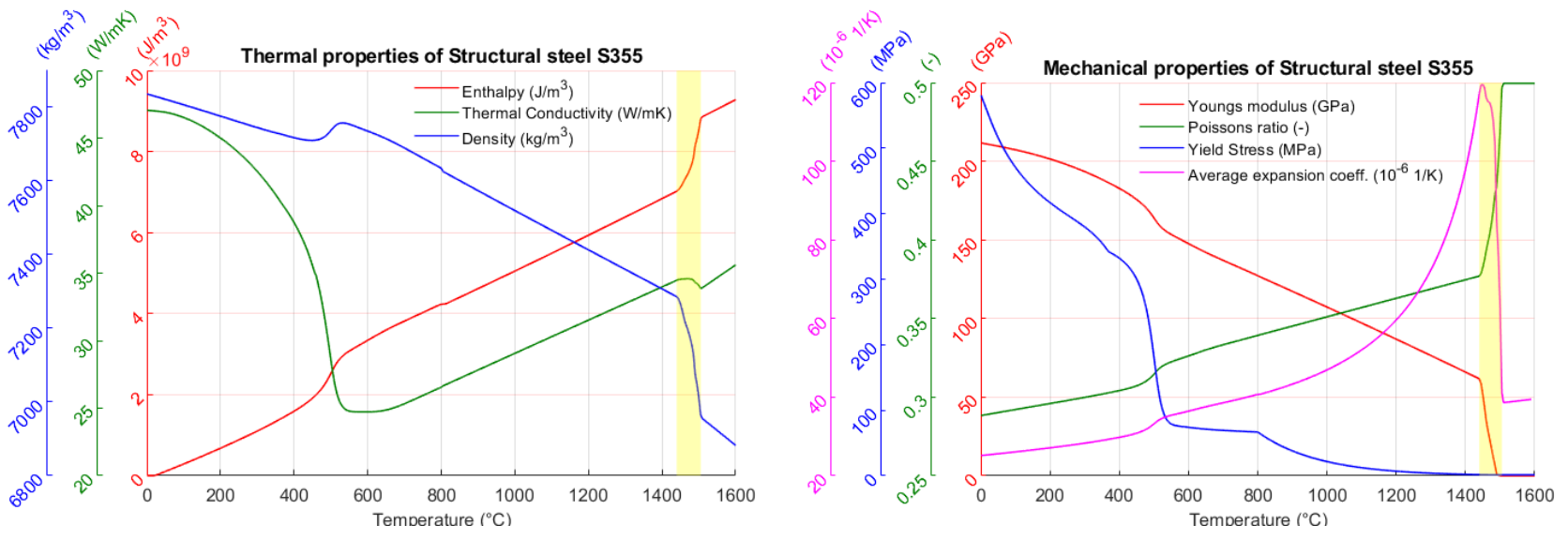

Figure 2 Thermophysical (left) and mechanical (right) properties of structural steel grade S355 
time-consuming experimental investigations. From this package one can obtain varying thermophysical and mechanical properties such as the enthalpy, the density, the thermal conductivity, the contraction, the Young's modulus, the Poisson's ratio, the yield stress, the ultimate strength, etc. in the whole temperature range. The properties for steel grade S355 obtained from this package are depicted in Figure 2.

\section{2.5D SLICE-THERMO-MECHANICAL MODEL}

The goal is to provide a coupled thermo-mechanical 2D analysis for a slice perpendicular to the strand axis, see Figure 1. The mathematical formulation of heat transfer and solidification to the temperature distribution and solid shell profile prediction in the BrDSM model is based on the governing equation of transient heat conduction. The 3D Fourier-Kirchhoff equation [7] was reformulated to the 2.5D-slice model to allows fast real time computation. This step neglects the heat transfer in the casting direction as well the convection term. The heat transfer equation is formulated based on the enthalpy approach, see [1] where the thermal solidification model with its boundary conditions is described in detail.

The most important source of mechanical stress is the thermal loading of the solidified material, followed by phase changes and extreme thermal gradients near the surface, exposed to intensive spray cooling and thermal contact with cooled rollers. With the knowledge of temperature field evolution and mechanical properties as a function of temperature according to Figure 2, the stress and strain evolution are coupled to the temperature according to the constitutive equation [8]

$$
\dot{\varepsilon}=\dot{\varepsilon}^{e l}+\dot{\varepsilon}^{v p}+\dot{\varepsilon}^{t h}=\frac{1+v}{E} \dot{\sigma}-\frac{v}{E} \operatorname{tr}(\dot{\sigma}) I+\frac{3}{2 \sigma_{e q}}\left[\frac{\sigma_{e q}-\sigma_{0}}{K \varepsilon_{e q}}\right]^{\frac{1}{m}} s+\alpha \dot{T} I
$$

The strain rate tensor $\dot{\varepsilon}$ is split into the elastic $\dot{\varepsilon}^{e l}$, viscoplastic $\dot{\varepsilon}^{v p}$ and thermal part $\dot{\varepsilon}^{\text {th }}$. The elastic component is described by Hooke's law in the equation (1) and is related to temperature-dependent material parameters: The Young's modulus $E$ and the Poisson coefficient $v$. The equivalent stress in the viscoplastic part of equation (9) can be given by

$\sigma_{e q}=\sigma_{0}+K \varepsilon_{e q}^{n} \dot{\varepsilon}_{e q}^{m}$

$\sigma_{0}(\mathrm{~Pa})$ denotes the static yield stress below which no viscoplastic deformation occurs, $K[\mathrm{~Pa}]$ is the so-called consistency of the material, $n(-)$ is the strain hardening coefficient, and $m(-)$ is the strain rate sensitivity coefficient. All these parameters are temperature-dependent. For the liquid and mushy state, the yield stress drops to zero, for the solid material it grows up as indicated in Figure 2 and later dominates the stress evolution in the cooled solid material. The stress deviator $s(\mathrm{~Pa})$ can be defined as

$s=\sigma+p I ; \quad p=-\frac{1}{3} \operatorname{tr} \sigma$

where $\sigma(\mathrm{Pa})$ is the Cauchy stress tensor and $p(\mathrm{~Pa})$ is the associated hydrostatic pressure. The Von Mises equivalent strain rate is defined as

$\dot{\varepsilon}_{e q}=\sqrt{\frac{2}{3} \dot{\varepsilon}_{i j}^{v p} \dot{\varepsilon}_{i j}^{v p}}$

and the von Mises equivalent stress $\sigma_{e q}$ is defined as

$\sigma_{e q}=\sqrt{\frac{3}{2} s_{i j} s_{i j}}$.

The other variables in the last term of equation (1) are the temperature-dependent thermal expansion coefficient $\alpha\left(\mathrm{K}^{-1}\right)$ and the temperature rate $\dot{T}(\mathrm{~K}), I$ is the identity tensor. The solution of the above presented equations represents a complex problem, especially due to coupling of the thermo-mechanical phenomena. 


\section{COMPUTATION AND RESULTS}

The whole casting strand is divided into a number of pieces (slices) of a defined thickness. In this work we decided to set the slice thickness to $2.5 \mathrm{~cm}$. The thickness is chosen wisely as the boundary conditions must describe heat withdrawal with a considerable precision (if the slice is thicker the precise boundary conditions will not be applied). The initial slice starts at the meniscus in the mould and moves downwards through the whole caster until it reaches the cutting part of the CCM. The explicit finite difference method in the thermal solidification model and the finite element method in mechanical stress-strain model are used. Thus, the numerical time step in thermal model is defined to avoid numerical oscillations in the explicit calculation. The temperature field in each slice is initially set to the temperature field of the previous slice and boundary conditions applied in this location are evaluated for the actual temperature field and last for a defined number of evaluations until the time required to move the slice to the following position is reached. Due to the $y-z$ planar symmetry, only one half of the slice is solved in each solution step The computation of thermal stress follows the strategy of the analysis of the temperature, where each solution step corresponds to the movement at the distance of one thickness of the slice, reading the appropriate input temperature of that position. Due to the inherent nonlinearity of the problem, a number of iterations must be performed within each step. The evolution of all necessary state parameters is recorded during the whole process.

Two models of temperature and mechanical stress-strain distributions were developed and adopted to a radial CCM of $27.32 \mathrm{~m}$ length, with the mould length of $1000 \mathrm{~mm}$ and the radius of caster $8.9 \mathrm{~m}$. The calculations were set for a billet $150 \times 150 \mathrm{~mm}$ with $\mathrm{SC}$ and $\mathrm{RC}$ of radius $\mathrm{R} 25 \mathrm{~mm}$. Due to the mentioned symmetry and for better visibility of the results only corners at the large radius (LR) side further from the center of the caster radius and the points at the middle of LR side surface are depicted in Figure 3 (left). The middle points of the LR side exhibit similar temperature profile throughout the whole caster. In contrast, the corner points differ at the end of primary cooling zone by $61^{\circ} \mathrm{C}$ from the rounded billet (RB). The billet with RC is less subcooled than
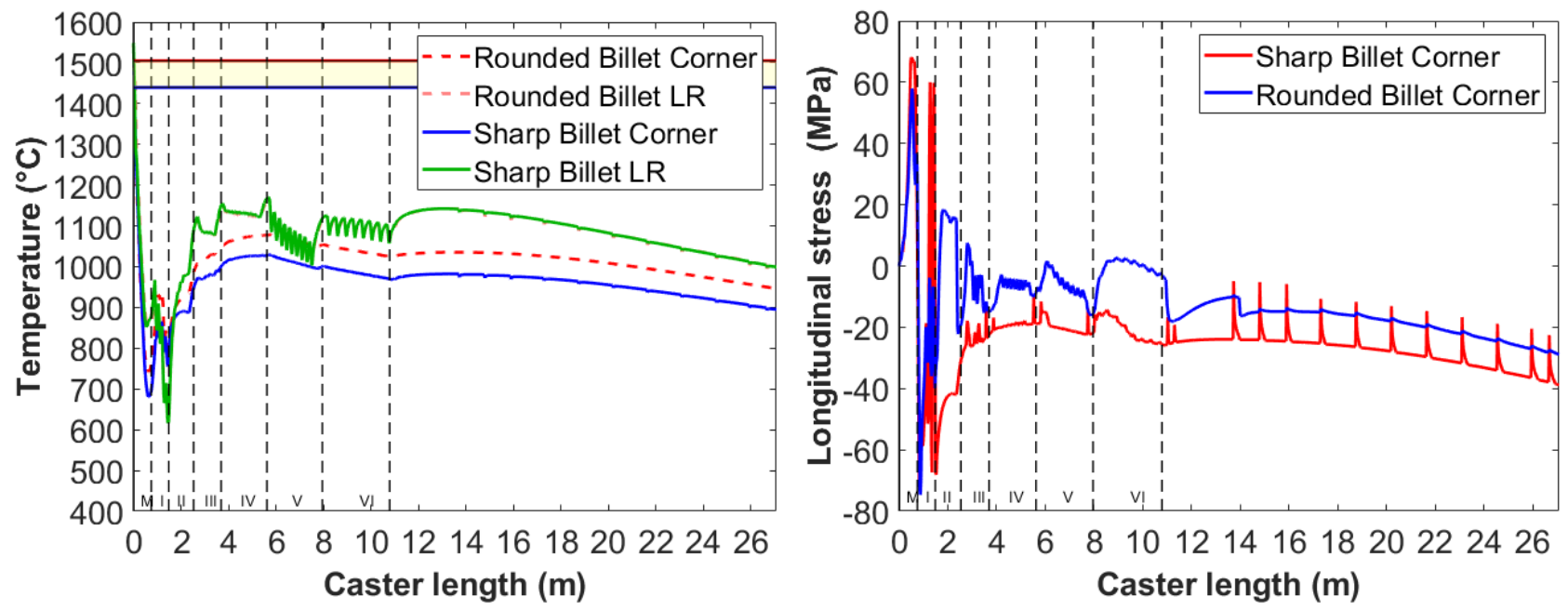

Figure 3 Temperature profile (left) and longitudinal stress component history (right) of SB and RB of steel grade S355

the sharp billet (SB). The temperature profile difference of the corners remains at around $50^{\circ} \mathrm{C}$ throughout the whole casting until the cutting zone. The evolution of longitudinal stress in the corners at the LR side are depicted in Figure 3 (right). Except of the mould exit, corner at the LR side of the RB exhibits relatively modest history with less extremes compared to the SB corner. Those sharp stress peaks in the primary section of the secondary cooling zone in both corner shapes are evoked in particular by intensive nozzle cooling nevertheless rollers induce excess stress as well which can be seen especially in case of SB after the end of the nozzle cooling section from 12th meter of the caster length. Figure 4 depicts the distribution of longitudinal 
stress component over the billet cross section in different locations. Especially before and immediately after the straightening section and at the end of the analysed time interval of the casting near the cutting section. The maximal tensile stress on the surface, which is dangerous for possible crack initiation, is reached during the straightening stage at the SB corner. For the RB, the tensile values are lower but exhibit similar behavior as the SB.
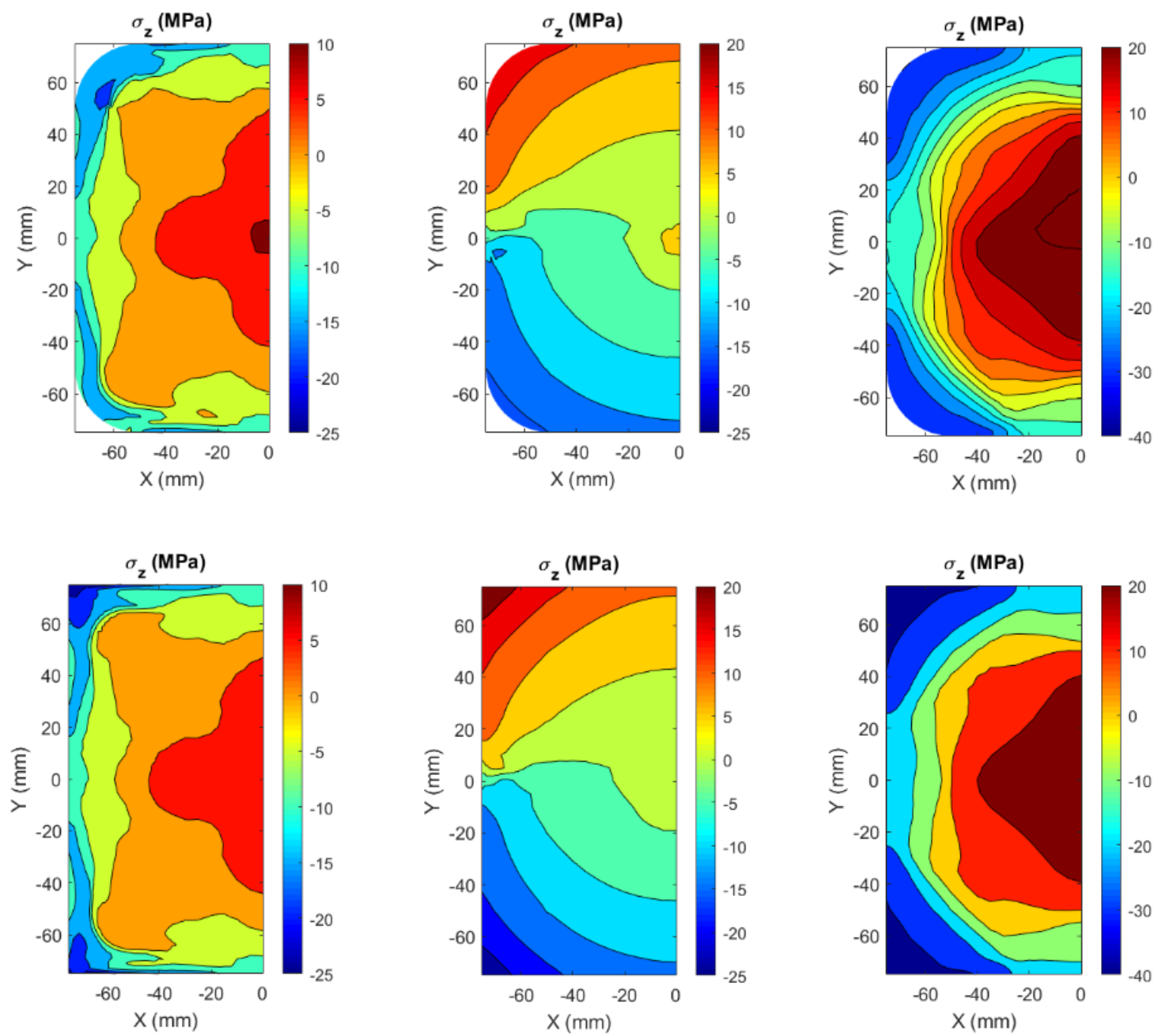

Figure 4 Longitudinal stress distribution in SB (bottom) and RB (top) of steel grade S355 before (left), after (middle) straightening and at the cutting section of the CCM (right)

Calculated values can be then substituted into the criteria and compared with the quality reports. The criteria optimization consists in obtaining the same quality results in both quality reports and prediction results based on the values of criteria gained from the models [9]. A number of criteria related to various quality parameters of steel exist. Some criteria are discussed in [10] and those were proposed and used for optimization of free defect casting. The rapid simulation of thermal and mechanical models can be improved by means of GPU techniques, which provide the fast evaluation of temperature and stress-strain distributions with a subsequent assessment of the strand quality based on the crack criteria. If none of the critical conditions is violated, the strand can be considered as a strand without quality issues and can be immediately used and processed.

\section{CONCLUSION}

In this work the 2.5D slice-thermo-mechanical model was created. In terms of thermal distribution, the previously developed 3D BrDSM was degraded to moving 2.5D slice model. This degradation is of a great 
importance while the results do not vary and the required calculation time is significantly reduced compared to 3D BrDSM, while there no need of the recalculation of the whole temperature field in the strand in each time step. Thus, it will allow the cooperation of the thermal and the mechanical model with the possibility of the strand quality prediction in real time. The construction idea of 2.5D slice model was presented. The results of temperature distribution from thermal solidification model were used into the mechanical model to calculate mechanical stress-strain distributions in the billets. The comparison of SB and RB was set due to the problematic topic of corner cracks formation. Temperature history and longitudinal stress results confirmed better behavior of the RC billet against SC billet. The primary reliability of these models was proved, and the future work is focused on verification of crack criteria which can predict the probability defects occurrence.

\section{ACKNOWLEDGEMENTS}

This work was supported by the Czech Science Foundation under contract No. 19-20802S "A coupled real-time thermo-mechanical solidification model of steel for crack prediction." and by the project of Brno University of Technology FSI-S-20-6295.

\section{REFERENCES}

[1] MAUDER, T., STETINA, J. High quality steel casting by using advanced mathematical methods. Metals. 2018, vol. 8, no. 12, doi: 10.3390/met8121019.

[2] THOMAS, B. G. Review on Modeling and Simulation of Continuous Casting. Steel Research International. 2017, vol. 1700312, p. 1700312.

[3] KIM, K., YEO, T. J., OH, K. H., and LEE, D. N. Effect of -Carbon and Sulfur in Continuously Cast Strand on Longitudinal Surface Cracks. ISIJ International. 1996, vol. 36, no. 3, pp. 284-289.

[4] YU, S., LONG, M., WANG, Q., CHEN, D. Effect of the strand corner structure on the corner stress during the bending and straightening processes in slab continuous casting. Journal of Manufacturing Processes. 2019, vol. 48, no. October, pp. 270-282.

[5] APPLIQU, S. and ANN, P. 2D1/2 thermal-mechanical model of continuous casting of steel using finite element method, 2003.

[6] MIETTINEN, J., LOUHENKILPI, S., KYTÖNEN, H. and LAINE, J,. IDS: Thermodynamic-kinetic-empirical tool for modelling of solidification, microstructure and material properties. Mathematics and Computers in Simulation. 2010, vol. 80, issue 7, pp. 1536-1550. ISSN 03784754.

[7] STEFANESCU, D. M. Science and Engineering of Casting Solidification. Second Edition, New York: Springer Science, 2009, p. 402. ISBN 978-0-387-74609-8

[8] KORIC, S., HIBBELER, L. C., LIU, R., THOMAS, B. G. Multiphysics model of metal solidification on the continuum level. Numerical Heat Transfer, Part B: Fundamentals. 2010, vol. 58, no. 6, pp. 371-392.

[9] BREZINA, M., MAUDER, T., KLIMES, L., STETINA, J., NAVRAT, T., PETRUSKA, J. Coupled real-time thermomechanical solidification model of continuously cast steel. In METAL 2019: $28^{\text {th }}$ International Conference on Metallurgy and Materials. Ostrava: TANGER, 2019, pp. 123-128.

[10] KULKARNI, M.S., BABU, A. S. Managing quality in continuous casting process using product quality model and simulated annealing. Journal of Materials Processing Technology. 2005, vol. 166, no. 12, pp. 294-306. 